\title{
Distribution of metals exposure and associations with cardiometabolic risk factors in the "Modeling the Epidemiologic Transition Study"
}

Adrienne S Ettinger ${ }^{1 *}$, Pascal Bovet ${ }^{2,3}$, Jacob Plange-Rhule ${ }^{4,5}$, Terrence E Forrester ${ }^{6}$, Estelle V Lambert ${ }^{7}$, Nicola Lupoli ${ }^{8}$, James Shine ${ }^{8}$, Lara R Dugas ${ }^{9}$, David Shoham ${ }^{9}$, Ramon A Durazo-Arvizu ${ }^{9}$, Richard S Cooper ${ }^{9}$ and Amy Luke ${ }^{9}$

\begin{abstract}
Background: Metals are known endocrine disruptors and have been linked to cardiometabolic diseases via multiple potential mechanisms, yet few human studies have both the exposure variability and biologically-relevant phenotype data available. We sought to examine the distribution of metals exposure and potential associations with cardiometabolic risk factors in the "Modeling the Epidemiologic Transition Study" (METS), a prospective cohort study designed to assess energy balance and change in body weight, diabetes and cardiovascular disease risk in five countries at different stages of social and economic development.
\end{abstract}

Methods: Young adults (25-45 years) of African descent were enrolled ( $N=500$ from each site) in: Ghana, South Africa, Seychelles, Jamaica and the U.S.A. We randomly selected 150 blood samples ( $N=30$ from each site) to determine concentrations of selected metals (arsenic, cadmium, lead, mercury) in a subset of participants at baseline and to examine associations with cardiometabolic risk factors.

Results: Median (interquartile range) metal concentrations ( $\mu \mathrm{g} / \mathrm{L}$ ) were: arsenic 8.5 (7.7); cadmium 0.01 (0.8); lead 16.6 (16.1); and mercury 1.5 (5.0). There were significant differences in metals concentrations by: site location, paid employment status, education, marital status, smoking, alcohol use, and fish intake. After adjusting for these covariates plus age and sex, arsenic (OR 4.1, 95\% C.I. 1.2, 14.6) and lead (OR 4.0, 95\% C.I. 1.6, 9.6) above the median values were significantly associated with elevated fasting glucose. These associations increased when models were further adjusted for percent body fat: arsenic (OR 5.6, 95\% C.I. 1.5, 21.2) and lead (OR 5.0, 95\% C.I. 2.0, 12.7). Cadmium and mercury were also related with increased odds of elevated fasting glucose, but the associations were not statistically significant. Arsenic was significantly associated with increased odds of low HDL cholesterol both with (OR 8.0, 95\% C.I. 1.8, 35.0) and without (OR 5.9, 95\% C.I. 1.5, 23.1) adjustment for percent body fat.

Conclusions: While not consistent for all cardiometabolic disease markers, these results are suggestive of potentially important associations between metals exposure and cardiometabolic risk. Future studies will examine these associations in the larger cohort over time.

Keywords: Arsenic, Cadmium, Cardiometabolic, Diabetes, Endocrine disruption, Lead, Mercury, Metals exposure, Obesity, Risk factor

\footnotetext{
* Correspondence: adrienne.ettinger@yale.edu

${ }^{1}$ Yale Center for Perinatal, Pediatric \& Environmental Epidemiology,

Department of Chronic Disease Epidemiology, Yale School of Public Health,

1 Church St, New Haven, CT 06510, USA

Full list of author information is available at the end of the article
} 


\section{Background}

There is increasing evidence that environmental chemicals may be contributing to the risk of diabetes [1], obesity [2], and cardiovascular disease [3]. Approximately 40\% of adults in the U.S. have diabetes or pre-diabetes [4] and about 70\% of this risk can be attributed to obesity $[5,6]$ which is due, in part, to underlying genetics, nutrition, and lifestyle factors. Almost 35\% of adults in the U.S. are obese [7] and, worldwide, the prevalence of obesity and of diabetes are increasing at alarming rates in industrialized and developing countries alike [8]. However, the rapid rise in obesity rates may not be explained solely by genetics and lifestyle factors. Environmental toxicants have been implicated as "obesogens" $[9,10]$ and risk factors for diabetes [11,12] and cardiovascular disease [3]. Numerous questions remain, however, regarding the timing of critical windows of exposure, susceptible populations at risk, and biological mechanisms of effect.

Toxic metals (arsenic, cadmium, lead, mercury) are ubiquitous environmental contaminants that can cause a wide range of adverse health effects in humans [13,14]. Metals have been identified as endocrine disruptors $[15,16]$ and, as such, potential etiologic factors with respect to diabetes. Exposure to metals may result in abnormal glucose metabolism, in turn increasing the risk of developing diabetes, through several plausible mechanisms [11], such as the induction of oxidative stress or interference in signal transduction or gene expression, resulting in beta-cell dysfunction and insulin resistance $[17,18]$. Metals have also been implicated in influencing estrogen receptor-mediated signaling [19] and may disrupt the glucocorticoid receptor [20], which regulates a wide range of biological processes in humans, including insulin sensitivity. It is also possible that reverse causality is at play, i.e., the existence of diabetes or pre-diabetes alters the metabolism of metals so that higher concentrations in the body result. Experimental evidence, however, suggests that insulin resistance and oxidative stress can be induced by metals, providing biological plausibility to metals-induced diabetes [17].

Metals have also been implicated in the risk of cardiovascular disease [21] which itself is a leading cause of morbidity and mortality. The cardiovascular effects of metals are related to the increased oxidative stress and inflammation resulting from the high affinity of metals for binding sulfhydryl groups thereby reducing oxidative defense mechanisms [22]. In vivo and in vitro studies have shown that metals may exert these effects through increased thrombosis, vascular smooth muscle dysfunction, endothelial dysfunction, dyslipidemia, and immune and mitochondrial dysfunction [23]. In epidemiologic studies, metals have been associated with hypertension, impaired kidney function, and peripheral arterial disease as well as all-cause and cardiovascular mortality [24-27].

Metabolic syndrome, a clustering of physiologic abnormalities (central obesity, dyslipidemia, hyperglycemia, hypertension) and a precursor of diabetes and cardiovascular disease, has also been associated with metals exposures in cross-sectional studies [28,29]. Thus far, however, the human evidence is limited and there is little understanding of the underlying etiology. The underlying pathophysiology connecting the different aspects of metabolic syndrome is an area of active interest and research, but it is clear that adiposity and insulin resistance play an important role [30]. Adipose tissue, as an important endocrine organ that modulates metabolism, inflammation and endothelial function, has been recognized in the development of metabolic syndrome, type 2 diabetes, and cardiovascular disease [31] and the state of obesity, itself, is an independent risk factor for cardiovascular disease [32] and type 2 diabetes [33]. However, the existence of "metabolically healthy" obese and "metabolically obese" normal weight phenotypes (31.7\% of obese and $23.5 \%$ of normal weight adults in NHANES 1999-2004 [34]) indicate that there is considerable unexplained variation in these associations $[35,36]$ which is not explained by diet and physical activity [37]. Although, others have shown that there is no healthy pattern of increased weight [38]. Given the sheer magnitude of the obesity, type 2 diabetes, and cardiovascular disease epidemics, it is imperative to consider a potential role of non-traditional risk factors, such as environmental chemicals, to better understand their contribution to the global disease burden.

To date, it has been difficult to assess the cardiometabolic effects of environmental metals exposure in human populations given the lack of specific outcome measurements and control of potential confounding variables in existing observational data and the inherent difficulty of testing these hypotheses through clinical trials. Many studies have focused on body mass index (BMI) and there is little information on the biologically-relevant phenotypes of obesity (e.g. fat distribution, fat/lean mass percentages) or the mechanistic intermediate markers of metabolic syndrome.

The objectives of this study were to: i) examine the distribution of selected blood metals (arsenic, cadmium, lead, mercury) and ii) evaluate the independent associations of blood metals with clinical markers of cardiometabolic risk in a cohort of young adults of African descent living in five countries across a range of social and economic development. In addition to the extensive phenotype data and stored biological specimens already available on this large sample population, a major strength is the widespread geographic distribution of the study sites which may underlie largely different exposures across the sites.

\section{Methods}

\section{Study population}

The "Modeling the Epidemiologic Transition Study" (METS) is a prospective cohort study designed to assess the association between physical activity levels and relative 
weight, weight gain and diabetes and cardiovascular disease risk in five population-based samples at different stages of social and economic development [39]. Twenty-five hundred $(2,500)$ young adults, age $25-45$ years, were enrolled between January 2010 and September 2011 with 500 participants ( $50 \%$ male) from each of five sites: rural Ghana (Kumasi), urban South Africa (Cape Town), the island nation of Seychelles (Victoria), urban Jamaica (Kingston) and the suburban Chicago area (Maywood, IL) of the United States of America (U.S.A.). The populations sampled are all of African descent and represent a range of social and economic development as defined by the United Nations Human Development Index (HDI) 2011 [40]: Ghana (HDI $=0.541)$ as a lower "medium" HDI country, South Africa (0.619) as "medium", Jamaica (0.727) and Seychelles (0.773) as "high", and the U.S.A. (0.910) as "very high". The present study includes a subset of 150 individuals from the cohort with metals concentrations measured in randomly selected baseline blood samples.

Extensive baseline data collection included: anthropometrics, estimates of body composition by bioelectrical impedance analysis, blood pressure, physical activity/inactivity by accelerometry and the World Health Organization Global Physical Activity Questionnaire [41,42], dietary intake by two 24-hour recalls, smoking and alcohol consumption history, self- and family medical history, and measures of employment history, occupation, education and wealth using the World Bank's Core Welfare Indicators Questionnaire [43]. Fasting blood samples were collected and analyzed for glucose, lipids, C-reactive protein, and cystatin C. Yearly follow-up examinations include measurement of body weight, blood pressure and change in medical history. Participants have been examined annually for the past two-to-three years and will continue to be followed for assessment of change in body weight and composition and diabetes and cardiovascular disease risk. Exclusion criteria for METS included: individuals with obvious infectious diseases (including active malaria), HIV-positive individuals, pregnant or lactating women, and individuals with conditions which prevented them from engaging in normal day-to-day physical activities (such as severe osteo- or rheumatoid arthritis or lower extremity disability). The research protocol was approved by the $\mathrm{Hu}$ man Subjects Committees of the participating institutions.

\section{Exposure assessment}

We randomly selected 150 baseline samples from archived whole blood specimens ( $\mathrm{n}=30$ per site) to be measured for concentrations of arsenic, cadmium, lead, and mercury. Venous blood samples were collected in 6-ml capacity plastic lavender top hematology Vacutainer ${ }^{\text {Twx }}$ tubes with EDTA as an anticoagulant (BD \#367863, Becton Dickinson, Franklin Lakes, NJ) and mixed by inverting gently five times. Whole blood $(0.5 \mathrm{ml})$ was transferred to 2-ml polypropylene microcentrifuge tubes with screw caps (Fisherbrand ${ }^{\text {ma }} \# 02-682-558$, Fisher Scientific Company L.L.C., Pittsburgh, PA), stored at $-80^{\circ} \mathrm{C}$. Samples were shipped on dry ice to the Harvard School of Public Health Trace Metals Laboratory (Boston, MA) for analysis.

All samples were handled in a Class 10,000 clean room under a Class 100 clean hood. All glass and plastic ware were cleaned by soaking in $10 \% \mathrm{HNO}_{3}$ for 24 hours and rinsing several times with deionized water. Blood samples were weighed and digested in $1 \mathrm{ml}$ of ultrapure concentrated $\mathrm{HNO}_{3}$ acid for 24 hours and then diluted to $5 \mathrm{ml}$ with deionized water after the addition of $0.5 \mathrm{ml}$ of $30 \%$ ultrapure hydrogen peroxide. Samples were analyzed using a dynamic reaction cell-inductively coupled plasma mass spectrometer (DRC-ICP-MS, Elan 6100, Perkin Elmer, Norwalk, CT). A mixed solution of $50 \mathrm{ng} / \mathrm{ml}$ In (for cadmium), $50 \mathrm{ng} / \mathrm{ml} \mathrm{Lu}$ (for lead) and $150 \mathrm{ng} / \mathrm{ml} \mathrm{Te}$ (for arsenic) in $5 \% \mathrm{HNO}_{3}$ were used as the internal standards. This solution was mixed with calibration standards and samples on-line using a mixing tee and a mixing coil. Samples were analyzed by the external calibration method using seven standards with concentrations ranging from 0 to $10 \mathrm{ng} / \mathrm{ml}$. Quality control measures included: analysis of initial calibration verification standard, continuous calibration standard, procedural blanks, duplicate samples, spiked samples, National Institute of Standard and Technology Standard Reference Material (NIST SRM) 1643etrace elements in water and NIST SRM955b-lead in blood. Results given were the average of five replicate measurements. The limits of detection $(\mathrm{ng} / \mathrm{ml})$ in blood for this procedure are: arsenic (0.02); cadmium (0.01); and lead (0.01). Total mercury was analyzed using the Direct Mercury Analyzer 80 Tricell (Milestone Inc., Monroe, CT) with a detection limit equal to $0.0015 \mathrm{ng} / \mathrm{ml}$.

\section{Statistical analysis}

Participant characteristics were summarized by sex: overall and by site. Blood metals concentrations were summarized overall and by site for each metal and by participant characteristics. For metals levels less than the limit of detection (LOD), a random number between 0 and LOD was substituted according to a uniform distribution. Next, metals concentrations were summarized for subjects with and without each of 11 recognized cardiometabolic disease risk factors dichotomized using standard clinical cut-points. Metabolic syndrome is diagnosed based on abnormalities in specific clustered clinical cardiometabolic measures that are associated with increased risk for diabetes and cardiovascular disease [44]. However, there are no specific cutoffs for populations of African descent, so we used cutoffs values for Europeans as suggested by the International Diabetes Federation [45]. Logistic regression was used to describe associations between metals exposure (dichotomized at the median) and cardiometabolic risk factors and 
odds ratios (OR) with 95\% confidence intervals (CI) presented. The main covariates of interest were determined $a$ priori based on biological considerations (age, sex, smoking, alcohol use, fish intake, percent body fat, physical activity) and others (education, marital status, paid employment status, site location) included using statistical considerations if they were significant $(\mathrm{p}=0.1)$ in bivariate models.

Final models were adjusted for "core" variables including: age, sex, and site location. "Fully-adjusted" models additionally included: education, paid employment, marital status smoking, alcohol use, and fish intake. Since adiposity may be a mediator, modifier, or outcome as a result of exposure to environmental chemicals, we did not adjust our initial models for BMI or other markers of excess body fat. However, we next additionally adjusted "core" and "fully-adjusted" models for percent body fat. All analyses were performed using STATA ${ }^{\circ}$ version 11.2 (Copyright 1985-2009, StataCorp LP College Station, Texas USA).

\section{Results}

As a result of the parent (METS) study design, participant characteristics varied widely across sites (Table 1). Since baseline blood samples ( $=30$ per site) were chosen randomly, the distribution of participants by sex within each site is not uniform; however, overall there were 74 males (49\%) and 76 females (51\%) participants included with mean $( \pm \mathrm{SD})$ age of $34.7( \pm 6.0)$ and $35.2( \pm 6.2)$ years, respectively. Forty-nine percent $(\mathrm{N}=74)$ of participants were classified as overweight and $29 \%(\mathrm{~N}=44)$ as obese by BMI; similarly, $49 \%$ had signs of abdominal adiposity measured by sex-specific elevated waist circumference.

Median (interquartile range) metals concentrations $(\mu \mathrm{g} / \mathrm{L})$ were: arsenic 8.5 (7.7); cadmium 0.01 (0.8); lead 16.6 (16.1); and mercury 1.5 (5.0). Overall, metals were significantly correlated with each other, for example: arsenic was significantly correlated with: lead $(\rho=0.3, p<0.001)$, cadmium $(\rho=0.5, \mathrm{p}<0.00001)$, and mercury $(\rho=0.6, \mathrm{p}<$ $0.00001)$. However, the strengths of these correlations varied between sites with the highest correlations among the metals observed in Jamaica and the lowest correlations among the metals in the U.S. (data not shown).

Overall, almost $63 \%$ and over $15 \%$ of samples, respectively, for cadmium and mercury were below the limits of detection (Table 2). No samples had undetectable levels of arsenic and less than one percent had undetectable levels of lead. As expected, metals concentrations differed significantly by site location. Ghana had the highest median blood lead levels and the Seychelles had the highest median blood levels of arsenic and mercury. Twenty-three percent $(\mathrm{N}=7)$ of Ghanaian participants, representing $20 \%(\mathrm{~N}=2)$ and $25 \%(\mathrm{~N}=5)$ of men and women, respectively, had blood lead levels $>10 \mu \mathrm{g} / \mathrm{dL}$ and one (3\%) female participant had a blood lead level $>25 \mu \mathrm{g} / \mathrm{dL}$. Forty-three percent of Ghanaians had blood lead levels $>3.6 \mu \mathrm{g} / \mathrm{dL}$ (95th percentile of NHANES 2009-2010 levels for ages 20 years and above) [46] and 17\% of Seychellois were also above this threshold. Interestingly, one participant at each site in the U.S.A. and South Africa also exceeded the NHANES 95th percentile for blood lead while none in Jamaica did.

There were no significant differences in blood metals by age group or sex, although in both the U.S. and South African samples women tended to have lower lead levels than the men. There were also significant differences in metals concentrations by: employment status, education, marital status, smoking, alcohol use, and fish intake (Table 3). Those participants reporting paid employment in the previous month had higher blood metals concentrations than those who did not work and these differences were significantly significant for all of the metals, except lead, suggesting that occupation may be related to exposure. However, there were no significant differences by employment type: non-manual, skilled manual, or unskilled manual labor. Most participants (73\%) in this subsample of the cohort reported being "never smokers" and only $18 \%(N=27)$ reported being "current" $(\geq 1$ /day for at least one year) smokers. Among smokers, the mean $( \pm \mathrm{SD})$ number of cigarettes smoked per day was $6.9 \pm 4.2$ (range $1-20)$ and this did not differ significantly by location ( $\mathrm{p}=$ 0.84 ). As would be expected, cadmium levels were significantly higher among current or occasional smokers compared to ex- or never smokers ( $\mathrm{p}=0.008)$.

Table 4 shows unadjusted associations between metals and cardiometabolic risk factors. Less than $3 \%$ and $7 \%$ of participants in the sample, respectively, reported having diabetes or high blood pressure. However, $34 \%(\mathrm{~N}=51)$ had elevated fasting blood glucose levels and $16 \%(\mathrm{~N}=24)$ had elevated systolic and 15.3\% ( $\mathrm{N}=23)$ had elevated diastolic blood pressure by baseline clinical measurement. Additionally, 30\% ( $\mathrm{N}=45)$ had elevated triglyceride levels and more than $31 \%(\mathrm{~N}=47)$ had low HDL cholesterol while more than $67 \%(\mathrm{~N}=101)$ had elevated LDL cholesterol. Geometric mean $(95 \% \mathrm{CI})$ metals levels were higher in those participants with elevated fasting glucose. Arsenic and mercury levels were also significantly higher in those with elevated triglyceride to HDL ratios.

Adjusting for age, sex, and site location, arsenic (OR 3.0, 95\% C.I. 1.0, 8.6), cadmium (OR 2.1, 95\% C.I. 1.0, 4.2), lead (OR 3.3, 95\% C.I. 1.5, 7.1), and mercury (OR 2.0, 95\% C.I. $0.7,5.7)$ were associated with an increased odds of elevated fasting glucose (Table 5); these associations were stronger upon adjustment for additional covariates and including percent body fat (Table 6). Additionally, arsenic above the median value was associated with significantly increased odds low HDL cholesterol (OR 1.22, 95\% C.I. 1.07, 1.38). Mercury above the median was associated with increased odds for 10 of the 11 cardiometabolic risk factors, but none of these associations was statistically significant. While these results, and several other non-significant 
Table 1 Distribution of participant characteristics by sex: overall and by site location

\begin{tabular}{|c|c|c|c|c|c|c|c|}
\hline & $\begin{array}{c}\text { Overall } \\
\text { Mean } \pm \text { SD } \\
\text { (or } \%)\end{array}$ & $\begin{array}{c}\text { U.S.A. } \\
\text { Mean } \pm \text { SD } \\
\text { (or \%) }\end{array}$ & $\begin{array}{c}\text { South Africa } \\
\text { Mean } \pm \text { SD } \\
(\text { or } \%)\end{array}$ & $\begin{array}{c}\text { Ghana } \\
\text { Mean } \pm \text { SD } \\
\text { (or } \%)\end{array}$ & $\begin{array}{c}\text { Jamaica } \\
\text { Mean } \pm \text { SD } \\
\text { (or } \%)\end{array}$ & $\begin{array}{c}\text { Seychelles } \\
\text { Mean } \pm \text { SD } \\
\text { (or } \%)\end{array}$ & p-value* \\
\hline Male & $\mathrm{N}=74$ & $\mathrm{~N}=10$ & $\mathrm{~N}=15$ & $\mathrm{~N}=10$ & $\mathrm{~N}=24$ & $\mathrm{~N}=15$ & \\
\hline Age (years) & $34.7 \pm 6.0$ & $34.8 \pm 6.4$ & $33.7 \pm 6.0$ & $36.5 \pm 6.4$ & $34.5 \pm 6.4$ & $34.6 \pm 5.3$ & 0.9 \\
\hline Education (years) & $11.2 \pm 2.2$ & $12.7 \pm 1.4$ & $10.3 \pm 1.9$ & $9.2 \pm 2.4$ & $11.1 \pm 1.9$ & $12.8 \pm 1.7$ & $<0.0001$ \\
\hline Weight (kg) & $74.0 \pm 17.2$ & $89.1 \pm 20.2$ & $64.1 \pm 16.4$ & $59.3 \pm 6.0$ & $75.4 \pm 12.9$ & $81.2 \pm 15.3$ & $<0.0001$ \\
\hline Height (cm) & $172.8 \pm 7.4$ & $171.5 \pm 4.3$ & $166.4 \pm 6.1$ & $170.3 \pm 6.4$ & $177.7 \pm 6.5$ & $173.9 \pm 7.2$ & $<0.0001$ \\
\hline Body Mass Index $\left(\mathrm{kg} / \mathrm{m}^{2}\right)$ & $24.7 \pm 5.4$ & $30.4 \pm 7.2$ & $22.9 \pm 4.5$ & $20.5 \pm 2.2$ & $23.9 \pm 4.1$ & $26.9 \pm 4.9$ & $<0.0001$ \\
\hline Waist circumference $(\mathrm{cm})$ & $83.2 \pm 13.5$ & $93.5 \pm 20.6$ & $79.3 \pm 12.3$ & $74.2 \pm 5.4$ & $81.8 \pm 10.6$ & $88.8 \pm 11.5$ & 0.004 \\
\hline Fat Mass (kg) & $20.2 \pm 11.1$ & $31.5 \pm 13.6$ & $18.7 \pm 9.7$ & $10.1 \pm 5.7$ & $19.4 \pm 8.5$ & $22.4 \pm 10.9$ & $<0.0001$ \\
\hline Percent Body Fat (\%) & $25.9 \pm 9.2$ & $34.0 \pm 7.8$ & $27.7 \pm 8.1$ & $16.8 \pm 8.9$ & $24.8 \pm 8.2$ & $26.4 \pm 8.0$ & $<0.0001$ \\
\hline Overweight (BMI $\geq 25$ ) & 40.5 & 70.0 & 26.7 & 10.0 & 37.5 & 60.0 & 0.02 \\
\hline Obese (BMI $\geq 30$ ) & 14.9 & 60.0 & 6.7 & 0.0 & 4.2 & 20.0 & $<0.0001$ \\
\hline Employed & 74.3 & 60.0 & 26.7 & 90.0 & 87.5 & 100.0 & $<0.0001$ \\
\hline Married (or Living as Married) & 51.4 & 40.0 & 40.0 & 90.0 & 37.5 & 66.7 & 0.03 \\
\hline Smoking (ever) & 37.8 & 40.0 & 66.7 & 0.0 & 33.3 & 40.0 & 0.02 \\
\hline Alcohol ( $\geq 12$ drinks/year) & 68.9 & 60.0 & 40.0 & 40.0 & 83.3 & 100.0 & $<0.0001$ \\
\hline Fish Intake ( $\geq 1$ serving/day) & 55.4 & 20.0 & 20.0 & 80.0 & 62.5 & 86.7 & 0.0001 \\
\hline Physical Activity (<21.5 min/day) & 60.8 & 80.0 & 33.3 & 50.0 & 70.8 & 66.7 & 0.09 \\
\hline Female & $\mathrm{N}=76$ & $\mathrm{~N}=\mathbf{2 0}$ & $\mathrm{N}=15$ & $\mathrm{~N}=\mathbf{2 0}$ & $N=6$ & $\mathrm{~N}=15$ & \\
\hline Age (years) & $35.2 \pm 6.2$ & $37.1 \pm 5.9$ & $33.7 \pm 6.8$ & $33.2 \pm 6.5$ & $37.2 \pm 4.6$ & $36.0 \pm 5.8$ & 0.2 \\
\hline Education (years) & $11.0 \pm 4.3$ & $14.1 \pm 2.4$ & $9.7 \pm 3.4$ & $6.9 \pm 3.5$ & $10.3 \pm 1.8$ & $14.0 \pm 3.7$ & $<0.0001$ \\
\hline Weight (kg) & $74.8 \pm 20.5$ & $77.7 \pm 19.0$ & $81.6 \pm 24.3$ & $67.8 \pm 16.1$ & $91.2 \pm 32.2$ & $67.1 \pm 12.3$ & 0.03 \\
\hline Height (cm) & $161.5 \pm 7.5$ & $164.3 \pm 8.8$ & $160.1 \pm 5.9$ & $157.5 \pm 7.2$ & $165.8 \pm 8.3$ & $162.8 \pm 4.8$ & 0.02 \\
\hline Body Mass Index $\left(\mathrm{kg} / \mathrm{m}^{2}\right)$ & $28.7 \pm 7.5$ & $28.6 \pm 5.3$ & $31.9 \pm 9.5$ & $27.3 \pm 6.5$ & $33.3 \pm 12.1$ & $25.4 \pm 5.2$ & 0.07 \\
\hline Waist circumference $(\mathrm{cm})$ & $90.4 \pm 14.9$ & $90.9 \pm 14.7$ & $95.7 \pm 15.3$ & $88.5 \pm 13.3$ & $101.1 \pm 21.2$ & $82.6 \pm 10.2$ & 0.05 \\
\hline Fat Mass (kg) & $30.5 \pm 13.8$ & $32.3 \pm 11.5$ & $37.7 \pm 15.5$ & $25.3 \pm 12.0$ & $39.0 \pm 21.7$ & $24.6 \pm 8.5$ & 0.01 \\
\hline Percent Body Fat (\%) & $39.4 \pm 8.7$ & $40.9 \pm 6.8$ & $45.1 \pm 6.9$ & $35.9 \pm 10.3$ & $40.4 \pm 10.3$ & $35.8 \pm 6.8$ & 0.009 \\
\hline Overweight (BMI $\geq 25$ ) & 57.9 & 50.0 & 80.0 & 45.0 & 83.3 & 53.3 & 0.2 \\
\hline Obese (BMI $\geq 30$ ) & 43.4 & 50.0 & 46.7 & 45.0 & 50.0 & 26.7 & 0.7 \\
\hline Employed & 52.6 & 60.0 & 6.7 & 50.0 & 33.3 & 100.0 & $<0.0001$ \\
\hline Married (or Living as Married) & 47.4 & 20.0 & 53.3 & 65.0 & 50.0 & 53.3 & 0.06 \\
\hline Smoking (ever) & 15.8 & 40.0 & 6.7 & 0.0 & 33.3 & 6.7 & 0.002 \\
\hline Alcohol ( $\geq 12$ drinks/year) & 42.1 & 55.0 & 20.0 & 35.0 & 66.7 & 46.7 & 0.2 \\
\hline Fish Intake ( $\geq 1$ serving/day) & 51.3 & 40.0 & 13.3 & 75.0 & 33.3 & 80.0 & 0.0002 \\
\hline Physical Activity (<21.5 min/day) & 84.2 & 90.0 & 93.3 & 80.0 & 83.3 & 73.3 & 0.6 \\
\hline
\end{tabular}

${ }^{*} p$-value from analysis of variation by site.

associations, are in the hypothesized direction of increased risk, there were also some statistically significant (and nonsignificant) negative associations that do not support our hypotheses.

\section{Discussion}

These data, while not entirely consistent, are suggestive of potentially important associations between blood metals concentrations and cardiometabolic risk. In particular, a statistically significant relationship was observed between arsenic and lead exposure and increased risk for elevated fasting glucose which was stronger upon adjustment for adiposity. This is consistent with previously published cross-sectional studies examining these associations $[28,47,48]$. Additionally, arsenic was significantly associated with increased odds of low HDL cholesterol both 
Table 2 METS blood metals concentrations, overall and by site location

\begin{tabular}{|c|c|c|c|c|c|c|c|c|c|c|c|c|}
\hline & \multirow[b]{2}{*}{$\mathbf{N}$} & \multirow[b]{2}{*}{ GM } & \multirow[b]{2}{*}{ (95\% Cl) } & \multirow[b]{2}{*}{$\%<$ LOD* $^{*}$} & \multirow[b]{2}{*}{ Minimum ${ }^{* *}$} & \multicolumn{6}{|c|}{ Selected percentiles } & \multirow[b]{2}{*}{ Maximum } \\
\hline & & & & & & 25th & $(95 \% \mathrm{Cl})$ & 50th & $(95 \% \mathrm{Cl})$ & 75th & $(95 \% \mathrm{Cl})$ & \\
\hline \multicolumn{13}{|l|}{ Overall } \\
\hline Lead $(\mu \mathrm{g} / \mathrm{dL})$ & 150 & 1.55 & $(1.30,1.85)$ & 0.7 & 0.001 & 0.97 & $(0.76,1.09)$ & 1.66 & $(1.34,1.93)$ & 2.60 & $(2.25,2.98)$ & 31.82 \\
\hline Cadmium ( $\mu \mathrm{g} / \mathrm{L})$ & 150 & 0.03 & $(0.02,0.04)$ & 62.7 & 0.000004 & 0.004 & $(0.003,0.005)$ & 0.008 & $(0.007,0.009)$ & 0.83 & $(0.29,1.08)$ & 3.08 \\
\hline Arsenic ( $\mu \mathrm{g} / \mathrm{L})$ & 150 & 8.23 & $(7.50,9.02)$ & 0.0 & 2.51 & 4.92 & $(4.57,5.87)$ & 8.48 & $(7.62,10.07)$ & 12.76 & $(11.59,14.99)$ & 26.85 \\
\hline Mercury $(\mu \mathrm{g} / \mathrm{L})$ & 150 & 0.85 & $(0.52,1.37)$ & 15.3 & 0.001 & 0.22 & $(0.07,0.51)$ & 1.49 & $(1.01,2.23)$ & 5.31 & $(3.42,15.38)$ & 70.36 \\
\hline \multicolumn{13}{|l|}{ U.S.A. } \\
\hline Lead $(\mu \mathrm{g} / \mathrm{dL})$ & 30 & 0.80 & $(0.61,1.05)$ & 0.0 & 0.22 & 0.44 & $(0.36,0.68)$ & 0.74 & $(0.47,1.14)$ & 1.28 & $(0.95,1.89)$ & 6.05 \\
\hline Cadmium ( $\mu \mathrm{g} / \mathrm{L})$ & 30 & 0.15 & $(0.07,0.31)$ & 26.7 & 0.005 & 0.009 & $(0.006,0.20)$ & 0.29 & $(0.14,0.60)$ & 0.83 & $(0.36,1.03)$ & 1.21 \\
\hline Arsenic ( $\mu \mathrm{g} / \mathrm{L})$ & 30 & 3.97 & $(3.62,4.35)$ & 0.0 & 2.61 & 3.33 & $(3.02,3.55)$ & 3.83 & $(3.42,4.56)$ & 4.72 & $(3.94,5.24)$ & 8.47 \\
\hline Mercury $(\mu \mathrm{g} / \mathrm{L})$ & 30 & 0.07 & $(0.03,0.20)$ & 33.3 & 0.001 & 0.007 & $(0.002,0.07)$ & 0.15 & $(0.01,0.36)$ & 0.69 & $(0.22,1.22)$ & 24.46 \\
\hline \multicolumn{13}{|l|}{ South Africa } \\
\hline Lead ( $\mu \mathrm{g} / \mathrm{dL})$ & 30 & 1.45 & $(1.22,1.74)$ & 0.0 & 0.58 & 0.99 & $(0.82,1.23)$ & 1.42 & $(1.16,1.86)$ & 1.99 & $(1.71,2.74)$ & 3.79 \\
\hline Cadmium ( $\mu \mathrm{g} / \mathrm{L})$ & 30 & 0.00 & $(0.00,0.01)$ & 93.3 & 0.000004 & 0.002 & $(0.0006,0.003)$ & 0.004 & $(0.003,0.007)$ & 0.008 & $(0.005,0.01)$ & 1.10 \\
\hline Arsenic ( $\mu \mathrm{g} / \mathrm{L})$ & 30 & 5.65 & $(5.18,6.16)$ & 0.0 & 3.98 & 4.71 & $(4.32,5.11)$ & 5.43 & $(4.81,6.52)$ & 6.88 & $(5.84,7.71)$ & 9.52 \\
\hline Mercury $(\mu \mathrm{g} / \mathrm{L})$ & 30 & 0.05 & $(0.02,0.15)$ & 40.0 & 0.001 & 0.005 & $(0.001,0.03)$ & 0.09 & $(0.01,0.33)$ & 0.47 & $(0.15,0.97)$ & 35.10 \\
\hline \multicolumn{13}{|l|}{ Ghana } \\
\hline Lead $(\mu \mathrm{g} / \mathrm{dL})$ & 30 & 3.12 & $(1.53,6.38)$ & 3.3 & 0.001 & 2.02 & $(1.35,2.79)$ & 2.96 & $(2.14,3.96)$ & 5.90 & $(3.59,25.97)$ & 31.82 \\
\hline Cadmium ( $\mu \mathrm{g} / \mathrm{L})$ & 30 & 0.00 & $(0.00,0.01)$ & 100.0 & 0.00003 & 0.004 & $(0.001,0.005)$ & 0.006 & $(0.004,0.007)$ & 0.008 & $(0.006,0.009)$ & 0.01 \\
\hline Arsenic ( $\mu \mathrm{g} / \mathrm{L})$ & 30 & 11.17 & $(9.69,12.87)$ & 0.0 & 2.51 & 10.30 & $(7.47,11.11)$ & 11.68 & $(10.46,12.57)$ & 14.00 & $(12.31,15.61)$ & 20.70 \\
\hline Mercury $(\mu \mathrm{g} / \mathrm{L})$ & 30 & 1.56 & $(1.01,2.42)$ & 3.3 & 0.009 & 1.08 & $(0.73,1.42)$ & 1.87 & $(1.33,2.29)$ & 2.93 & $(2.14,4.13)$ & 11.25 \\
\hline \multicolumn{13}{|l|}{ Jamaica } \\
\hline Lead $(\mu \mathrm{g} / \mathrm{dL})$ & 30 & 0.96 & $(0.77,1.19)$ & 0.0 & 0.25 & 0.69 & $(0.44,0.95)$ & 1.01 & $(0.77,1.24)$ & 1.39 & $(1.06,2.01)$ & 2.57 \\
\hline Cadmium ( $\mu \mathrm{g} / \mathrm{L})$ & 30 & 0.01 & $(0.00,0.04)$ & 76.7 & 0.0002 & 0.004 & $(0.001,0.004)$ & 0.005 & $(0.00,0.01)$ & 0.17 & $(0.01,1.10)$ & 3.08 \\
\hline Arsenic ( $\mu \mathrm{g} / \mathrm{L})$ & 30 & 9.24 & $(8.37,10.19)$ & 0.0 & 6.35 & 7.89 & $(6.99,8.49)$ & 8.61 & $(8.09,9.65)$ & 10.23 & $(8.97,14.20)$ & 19.02 \\
\hline Mercury $(\mu \mathrm{g} / \mathrm{L})$ & 30 & 2.43 & $(1.82,3.25)$ & 0.0 & 0.22 & 1.66 & $(0.82,2.40)$ & 2.54 & $(2.19,3.60)$ & 3.84 & $(3.39,6.41)$ & 8.33 \\
\hline \multicolumn{13}{|l|}{ Seychelles } \\
\hline Lead ( $\mu \mathrm{g} / \mathrm{dL})$ & 30 & 2.56 & $(2.26,2.90)$ & 0.0 & 1.46 & 1.96 & $(1.72,2.43)$ & 2.49 & $(2.21,2.80)$ & 3.23 & $(2.60,3.92)$ & 6.71 \\
\hline Cadmium ( $\mu \mathrm{g} / \mathrm{L})$ & 30 & 0.47 & $(0.21,1.06)$ & 16.7 & 0.001 & 1.04 & $(0.01,1.15)$ & 1.17 & $(1.11,1.22)$ & 1.27 & $(1.19,1.42)$ & 1.56 \\
\hline Arsenic ( $\mu \mathrm{g} / \mathrm{L})$ & 30 & 16.29 & $(14.93,17.77)$ & 0.0 & 10.55 & 13.98 & $(11.93,15.56)$ & 16.38 & $(14.40,17.05)$ & 18.59 & $(16.59,23.10)$ & 26.85 \\
\hline Mercury $(\mu \mathrm{g} / \mathrm{L})$ & 30 & 28.47 & $(23.03,35.18)$ & 0.0 & 9.20 & 18.16 & $(13.70,24.45)$ & 28.34 & $(22.34,39.19)$ & 48.58 & $(31.77,60.84)$ & 70.36 \\
\hline
\end{tabular}

*Limits of detection (ng/ml): Lead (0.01); Cadmium (0.01); Arsenic (0.02); Mercury (0.0015).

**For levels less than LOD: substituted a random number between 0 and LOD (according to uniform distribution).

with and without adjustment for percent body fat. Mercury was associated with increased odds for 10 of the 11 cardiometabolic risk factors though these associations were not statistically significant. We propose to further evaluate these preliminary associations in the larger METS cohort of 2,500 individuals at baseline and also assess changes in risk over time taking advantage of the longitudinal followup of the cohort.

There is increasing concern that unintended exposures to environmental contaminants may be adversely affecting human health. The association between environmental contaminants and diabetes, obesity, and cardiovascular disease is an emerging area of interest in the field of environmental health sciences $[2,49]$. Despite the conventional focus on clinical and modifiable lifestyle risk factors, the incidence of cardiometabolic diseases continues to increase at alarming rates bringing the focus on environmental risk factors to the forefront. Simultaneously, there is increasing recognition that many chronic diseases have their origins in fetal life [50] and exposure to endocrine disrupting chemicals, such as metals, during critical windows of fetal development may be important contributing factors that "program" individuals to be at increased risk for metabolic disturbances by altering normal glycemic control or adipocyte differentiation [51,52]. Nonetheless, important questions remain about timing of critical 
Table 3 Geometric mean (95\% confidence interval) blood metals concentrations by participant characteristics

\begin{tabular}{|c|c|c|c|c|c|c|c|c|c|}
\hline & & Lead $(\mu \mathrm{g} / \mathrm{dL})$ & & Cadmium ( $\mu \mathrm{g} / \mathrm{L})$ & & Arsenic $(\mu \mathrm{g} / \mathrm{L})$ & & Mercury ( $\mu \mathrm{g} / \mathrm{L})$ & \\
\hline Variable & N (\%) & GM $(95 \%$ Cl) & p-value & GM $(95 \% \mathrm{Cl})$ & $\mathrm{p}$-value & GM $(95 \%$ Cl) & $p$-value & GM $(95 \%$ Cl) & $\mathrm{p}$-value \\
\hline \multicolumn{10}{|l|}{ Site Location } \\
\hline U.S.A. & $30(20)$ & $0.80(0.61,1.05)$ & $<0.00001$ & $0.15(0.07,0.31)$ & $<0.00001$ & $3.97(3.62,4.35)$ & $<0.00001$ & $0.07(0.03,0.20)$ & $<0.00001$ \\
\hline South Africa & $30(20)$ & $1.45(1.22,1.74)$ & & $0.00(0.00,0.01)$ & & $5.65(5.18,6.16)$ & & $0.05(0.02,0.15)$ & \\
\hline Ghana & $30(20)$ & $3.12(1.53,6.38)$ & & $0.00(0.00,0.01)$ & & $11.17(9.69,12.87)$ & & $1.56(1.01,2.42)$ & \\
\hline Jamaica & $30(20)$ & $0.96(0.77,1.19)$ & & $0.01(0.00,0.04)$ & & $9.24(8.37,10.19)$ & & $2.43(1.82,3.25)$ & \\
\hline Seychelles & $30(20)$ & $2.56(2.26,2.90)$ & & $0.47(0.21,1.06)$ & & $16.29(14.93,17.77)$ & & $28.47(23.03,35.18)$ & \\
\hline \multicolumn{10}{|l|}{ Age group } \\
\hline$<35$ years & $72(48)$ & $1.46(1.07,1.99)$ & 0.64 & $0.02(0.01,0.04)$ & 0.09 & $8.13(7.17,9.22)$ & 0.53 & $0.81(0.41,1.61)$ & 0.22 \\
\hline$\geq 35$ years & $78(52)$ & $1.64(1.35,1.99)$ & & $0.04(0.02,0.07)$ & & $8.32(7.26,9.53)$ & & $0.88(0.44,1.75)$ & \\
\hline \multicolumn{10}{|l|}{ Sex } \\
\hline Male & $74(49)$ & $1.68(1.41,2.00)$ & 0.35 & $0.03(0.02,0.06)$ & 0.43 & $8.94(7.93,10.07)$ & 0.21 & $1.61(0.88,2.94)$ & 0.13 \\
\hline Female & $76(51)$ & $1.44(1.05,1.96)$ & & $0.02(0.01,0.05)$ & & $7.59(6.59,8.73)$ & & $0.45(0.22,0.94)$ & \\
\hline \multicolumn{10}{|l|}{ Marital Status } \\
\hline Married/living as married & $74(49)$ & $1.97(1.57,2.48)$ & 0.02 & $0.02(0.01,0.04)$ & 0.38 & $9.48(8.39,10.71)$ & 0.007 & $1.40(0.74,2.65)$ & 0.08 \\
\hline Not married* & $76(51)$ & $1.22(0.94,1.60)$ & & $0.03(0.02,0.06)$ & & $7.17(6.28,8.18)$ & & $0.52(0.25,1.06)$ & \\
\hline \multicolumn{10}{|l|}{ Education } \\
\hline$\leq 11$ years & $83(55)$ & $1.84(1.39,2.44)$ & 0.03 & $0.01(0.01,0.02)$ & 0.03 & $9.09(8.20,10.09)$ & 0.23 & $0.90(0.51,1.60)$ & 0.004 \\
\hline$>11$ years & $67(45)$ & $1.25(1.03,1.52)$ & & $0.07(0.04,0.14)$ & & $7.27(6.20,8.52)$ & & $0.78(0.35,1.78)$ & \\
\hline \multicolumn{10}{|l|}{ Paid Employment (past month) } \\
\hline Yes & $95(63)$ & $1.71(1.42,2.06)$ & 0.81 & $0.05(0.03,0.09)$ & $<0.00001$ & $9.63(8.60,10.79)$ & $<0.00001$ & $2.24(1.33,3.80)$ & $<0.00001$ \\
\hline No & $55(37)$ & $1.31(0.90,1.90)$ & & $0.01(0.00,0.02)$ & & $6.26(5.48,7.15)$ & & $0.16(0.07,0.34)$ & \\
\hline \multicolumn{10}{|l|}{ Employment type } \\
\hline Non-manual labor & $37(25)$ & $1.07(0.78,1.45)$ & 0.12 & $0.10(0.04,0.22)$ & 0.22 & $7.18(5.67,9.09)$ & 0.38 & $0.76(0.25,2.32)$ & 0.22 \\
\hline Skilled manual labor & $16(11)$ & $1.39(1.03,1.88)$ & & $0.03(0.01,0.13)$ & & $7.73(6.08,9.81)$ & & $0.63(0.13,3.12)$ & \\
\hline Unskilled manual labor & $92(61)$ & $1.87(1.45,2.41)$ & & $0.02(0.01,0.03)$ & & $9.02(8.12,10.03)$ & & $1.10(0.63,1.93)$ & \\
\hline Missing & $5(3)$ & $1.01(0.45,2.31)$ & & $0.02(0.00,0.35)$ & & $5.01(2.77,9.09)$ & & $0.04(0.00,2.76)$ & \\
\hline \multicolumn{10}{|l|}{ Cigarette smoking } \\
\hline Smoker ( $\geq 1 /$ day for year) & $27(18)$ & $1.58(1.28,1.96)$ & 0.67 & $0.13(0.04,0.37)$ & 0.0008 & $6.34(5.12,7.85)$ & 0.03 & $0.20(0.06,0.74)$ & 0.05 \\
\hline Occasional smoker & $8(5)$ & $2.22(1.59,3.10)$ & & $0.05(0.00,1.30)$ & & $12.09(8.03,18.20)$ & & $5.96(0.56,63.89)$ & \\
\hline Ex-smoker (for at least 1 year) & $5(3)$ & $1.61(0.61,4.26)$ & & $0.01(0.00,0.27)$ & & $8.79(5.68,13.60)$ & & $2.21(0.23,21.52)$ & \\
\hline Never-smoker & $110(73)$ & $1.50(1.18,1.90)$ & & $0.02(0.01,0.03)$ & & $8.50(7.63,9.47)$ & & $1.00(0.59,1.71)$ & \\
\hline
\end{tabular}


Table 3 Geometric mean (95\% confidence interval) blood metals concentrations by participant characteristics (Continued)

\begin{tabular}{|c|c|c|c|c|c|c|c|c|c|}
\hline \multicolumn{10}{|l|}{ Alcohol Use ( $\geq 12$ drinks/year) } \\
\hline Yes & $83(55)$ & $1.42(1.10,1.84)$ & 0.06 & $0.05(0.03,0.09)$ & 0.002 & $8.94(7.88,10.15)$ & 0.031 & $1.59(0.84,3.01)$ & 0.006 \\
\hline No/Missing & $67(45)$ & $1.72(1.34,2.22)$ & & $0.01(0.01,0.02)$ & & $7.41(6.49,8.47)$ & & $0.39(0.19,0.78)$ & \\
\hline \multicolumn{10}{|l|}{ Fish Intake ( $\geq 1$ serving/day) } \\
\hline Yes & $80(53.3)$ & $2.13(1.72,2.65)$ & 0.003 & $0.46(0.02,0.09)$ & 0.0009 & $10.59(9.40,11.93)$ & $<0.00001$ & $2.85(1.72,4.72)$ & 0.0002 \\
\hline No & $70(46.7)$ & $1.07(0.82,1.41)$ & & $0.01(0.01,0.03)$ & & $6.16(5.52,6.88)$ & & $0.21(0.10,0.44)$ & \\
\hline \multicolumn{10}{|l|}{ Physical Activity } \\
\hline$<21.5$ mins/day & $109(73)$ & $1.39(1.11,1.75)$ & 0.62 & $0.03(0.02,0.05)$ & 0.98 & $7.87(7.02,8.82)$ & 0.36 & $0.79(0.44,1.41)$ & 0.68 \\
\hline$\geq 21.5 \mathrm{mins} /$ day & $41(27)$ & $2.06(1.61,2.64)$ & & $0.03(0.01,0.06)$ & & $9.25(7.98,10.73)$ & & $1.02(0.44,2.40)$ & \\
\hline \multicolumn{10}{|l|}{ Weight group } \\
\hline Underweight, $\mathrm{BMl}<18.5$ & $6(4)$ & $1.83(1.21,2.77)$ & 0.61 & $0.00(0.00,0.01)$ & 0.47 & $8.06(5.02,12.92)$ & 0.11 & $0.39(0.00,31.81)$ & 0.04 \\
\hline Normal weight, BMI 18.5 to $<25$ & $70(47)$ & $1.62(1.17,2.26)$ & & $0.03(0.02,0.06)$ & & $8.74(7.62,10.01)$ & & $0.91(0.48,1.75)$ & \\
\hline Overweight, BMI $\geq 25$ to $<30$ & $30(20)$ & $1.67(1.24,2.24)$ & & $0.01(0.00,0.05)$ & & $9.55(7.91,11.53)$ & & $2.38(0.86,6.59)$ & \\
\hline Obese, $\mathrm{BMI} \geq 30$ & $44(29)$ & $1.34(1.04,1.73)$ & & $0.04(0.02,0.09)$ & & $6.77(5.66,8.10)$ & & $0.41(0.16,1.09)$ & \\
\hline
\end{tabular}

*Not married includes: widowed, divorced, separated, and never married. 
Table 4 Unadjusted associations between metals exposures and cardiometabolic risk factors

\begin{tabular}{|c|c|c|c|c|c|c|c|c|c|}
\hline \multirow[b]{2}{*}{ Cardiometabolic risk factor } & \multirow[b]{2}{*}{ N (\%) } & \multirow{2}{*}{$\begin{array}{l}\text { Lead }(\mu \mathrm{g} / \mathrm{dL}) \\
\mathrm{GM}(95 \% \mathrm{Cl})\end{array}$} & \multicolumn{3}{|c|}{ Cadmium ( $\mu \mathrm{g} / \mathrm{L})$} & \multirow{2}{*}{$\begin{array}{l}\text { Arsenic }(\mu \mathrm{g} / \mathrm{L}) \\
\mathrm{GM}(95 \% \mathrm{Cl})\end{array}$} & \multicolumn{3}{|c|}{ Mercury $(\mu \mathrm{g} / \mathrm{L})$} \\
\hline & & & p-value & GM $(95 \% \mathrm{Cl})$ & p-value & & p-value & GM $(95 \% \mathrm{Cl})$ & p-value \\
\hline \multicolumn{10}{|l|}{ Overweight, BMI $\geq 25$} \\
\hline Yes & $74(49.3)$ & $1.46(1.21,1.77)$ & 0.53 & $0.03(0.01,0.05)$ & 0.84 & $7.78(6.80,8.90)$ & 0.24 & $0.84(0.41,1.73)$ & 0.98 \\
\hline No & $76(50.7)$ & $1.64(1.21,2.22)$ & & $0.03(0.01,0.05)$ & & $8.68(7.64,9.87)$ & & $0.85(0.44,1.64)$ & \\
\hline \multicolumn{10}{|l|}{ Obese, BMI $\geq 30$} \\
\hline Yes & $44(29.3)$ & $1.34(1.04,1.73)$ & 0.31 & $0.04(0.02,0.09)$ & 0.18 & $6.77(5.66,8.10)$ & 0.008 & $0.41(0.16,1.09)$ & 0.06 \\
\hline No & $106(70.7)$ & $1.65(1.31,2.08)$ & & $0.02(0.01,0.04)$ & & $8.92(8.03,9.90)$ & & $1.14(0.66,1.97)$ & \\
\hline \multicolumn{10}{|l|}{$\begin{array}{l}\text { Waist Circumference } \geq 94 \mathrm{~cm} \\
\text { (males) or } \geq 80 \mathrm{~cm} \text { (females) }\end{array}$} \\
\hline Yes & $74(49.3)$ & $1.49(1.21,1.84)$ & 0.69 & $0.03(0.02,0.07)$ & 0.31 & $7.35(6.40,8.44)$ & 0.02 & $0.55(0.26,1.18)$ & 0.08 \\
\hline No & $76(50.7)$ & $1.61(1.20,2.15)$ & & $0.02(0.01,0.04)$ & & $9.18(8.15,10.34)$ & & $1.29(0.72,2.32)$ & \\
\hline \multicolumn{10}{|l|}{ Glucose $\geq 100$ mg/dL (fasting) } \\
\hline Yes & $51(34.0)$ & $2.30(1.78,2.98)$ & 0.002 & $0.06(0.03,0.14)$ & 0.009 & $9.70(8.19,11.48)$ & 0.01 & $1.62(0.70,3.76)$ & 0.06 \\
\hline No & $99(66.0)$ & $1.26(1.01,1.59)$ & & $0.02(0.01,0.03)$ & & $7.56(6.79,8.41)$ & & $0.61(0.34,1.08)$ & \\
\hline \multicolumn{10}{|l|}{ LDL cholesterol $\geq 2.59 \mathrm{mmol} / \mathrm{L}$} \\
\hline Yes & $101(67.3)$ & $1.51(1.28,1.77)$ & 0.66 & $0.03(0.02,0.06)$ & 0.11 & $8.42(7.56,9.38)$ & 0.47 & $1.25(0.71,2.21)$ & 0.02 \\
\hline No & $49(32.7)$ & $1.64(1.05,2.56)$ & & $0.02(0.01,0.03)$ & & $7.84(6.56,9.36)$ & & $0.38(0.16,0.90)$ & \\
\hline \multicolumn{10}{|l|}{ Triglycerides $\geq 1.7 \mathrm{mmol} / \mathrm{L}$} \\
\hline Yes & $45(30.0)$ & $1.14(0.90,1.46)$ & 0.05 & $0.02(0.01,0.04)$ & 0.37 & $8.43(7.33,9.68)$ & 0.73 & $1.13(0.59,2.17)$ & 0.44 \\
\hline No & $105(70.0)$ & $1.76(1.40,2.23)$ & & $0.03(0.02,0.05)$ & & $8.14(7.23,9.17)$ & & $0.75(0.40,1.41)$ & \\
\hline \multicolumn{10}{|l|}{$\begin{array}{l}\mathrm{HDL} \text { cholesterol }<1.03 \text { (males) } \\
\quad \text { or }<1.29 \text { (females) } \mathrm{mmol} / \mathrm{L}\end{array}$} \\
\hline Yes & $47(31.3)$ & $1.69(1.06,2.71)$ & 0.51 & $0.01(0.01,0.03)$ & 0.06 & $8.27(6.83,10.00)$ & 0.94 & $0.39(0.16,0.97)$ & 0.04 \\
\hline No & $103(68.7)$ & $1.49(1.27,1.74)$ & & $0.04(0.02,0.06)$ & & $8.21(7.39,9.11)$ & & $1.20(0.69,2.10)$ & \\
\hline \multicolumn{10}{|l|}{$\begin{array}{l}\text { Triglyceride/HDL ratio }>3.5 \\
\text { (males) or }>2.5 \text { (females) }\end{array}$} \\
\hline Yes & $55(36.7)$ & $1.41(1.17,1.71)$ & 0.44 & $0.04(0.02,0.08)$ & 0.27 & $10.69(9.55,11.97)$ & $<0.00001$ & $4.49(2.78,7.26)$ & $<0.00001$ \\
\hline No & $95(63.3)$ & $1.64(1.26,2.13)$ & & $0.02(0.01,0.04)$ & & $7.07(6.26,7.97)$ & & $0.32(0.17,0.61)$ & \\
\hline \multicolumn{10}{|l|}{ C-reactive protein $\geq 10 \mathrm{mg} / \mathrm{L}$} \\
\hline Yes & $43(28.7)$ & $1.14(0.93,1.40)$ & 0.06 & $0.01(0.01,0.03)$ & 0.03 & $8.79(7.78,9.92)$ & 0.37 & $1.30(0.66,2.54)$ & 0.27 \\
\hline No & $107(71.3)$ & $1.75(1.38,2.21)$ & & $0.04(0.02,0.06)$ & & $8.01(7.10,9.03)$ & & $0.71(0.38,1.32)$ & \\
\hline
\end{tabular}


Table 4 Unadjusted associations between metals exposures and cardiometabolic risk factors (Continued)

\begin{tabular}{|c|c|c|c|c|c|c|c|c|c|}
\hline \multicolumn{10}{|l|}{ Systolic $\geq 130 \mathrm{mmHg}$} \\
\hline Yes & $24(16.0)$ & $1.56(1.24,1.96)$ & 0.97 & $0.04(0.01,0.16)$ & 0.40 & $7.08(5.44,9.21)$ & 0.16 & $0.76(0.22,2.64)$ & 0.85 \\
\hline No & $126(84.0)$ & $1.55(1.25,1.91)$ & & $0.02(0.02,0.04)$ & & $8.46(7.67,9.34)$ & & $0.86(0.51,1.46)$ & \\
\hline \multicolumn{10}{|l|}{ Diastolic $\geq 85 \mathrm{mmHg}$} \\
\hline Yes & $23(15.3)$ & $1.69(1.23,2.32)$ & 0.68 & $0.03(0.01,0.14)$ & 0.63 & $6.26(4.80,8.16)$ & 0.02 & $0.49(0.14,1.77)$ & 0.35 \\
\hline No & $127(84.7)$ & $1.53(1.24,1.87)$ & & $0.03(0.02,0.04)$ & & $8.64(7.85,9.52)$ & & $0.93(0.55,1.57)$ & \\
\hline
\end{tabular}


Table 5 Adjusted* associations between metals (dichotomized at median) and cardiometabolic risk factors

\begin{tabular}{|c|c|c|c|c|c|c|c|c|c|}
\hline \multirow[b]{2}{*}{ Cardiometabolic risk factor } & \multirow[b]{2}{*}{$\mathrm{N}(\%)$} & \multicolumn{2}{|c|}{ Lead $(>1.66 \mu \mathrm{g} / \mathrm{dL})$} & \multicolumn{2}{|c|}{ Cadmium $(>0.008 \mu \mathrm{g} / \mathrm{L})$} & \multicolumn{2}{|c|}{ Arsenic $(>8.48 \mu \mathrm{g} / \mathrm{L})$} & \multicolumn{2}{|c|}{ Mercury $(>1.49 \mu \mathrm{g} / \mathrm{L})$} \\
\hline & & $\begin{array}{l}\text { Core model } \\
\text { OR }(95 \% \mathrm{Cl})^{*}\end{array}$ & $\begin{array}{l}\text { Fully-adjusted } \\
\text { OR }(95 \% \mathrm{Cl})^{* *}\end{array}$ & $\begin{array}{l}\text { Core model } \\
\text { OR }(95 \% \mathrm{Cl})^{*}\end{array}$ & $\begin{array}{l}\text { Fully-adjusted } \\
\text { OR }(95 \% \mathrm{Cl})^{* *}\end{array}$ & $\begin{array}{l}\text { Core model } \\
\text { OR }(95 \% \mathrm{Cl})^{*}\end{array}$ & $\begin{array}{l}\text { Fully-adjusted } \\
\text { OR }(95 \% \mathrm{Cl})^{* *}\end{array}$ & $\begin{array}{l}\text { Core model } \\
\text { OR }(95 \% \mathrm{Cl})^{*}\end{array}$ & $\begin{array}{l}\text { Fully-adjusted } \\
\text { OR }(95 \% \mathrm{Cl})^{* *}\end{array}$ \\
\hline Overweight, BMI $\geq 25$ & $74(49.3)$ & $0.51(0.24,1.08)$ & $0.60(0.27,1.36)$ & $0.84(0.43,1.64)$ & $0.71(0.33,1.52)$ & $0.27(0.10,0.77)$ & $0.27(0.09,0.81)$ & $1.68(0.61,4.63)$ & $1.73(0.61,4.91)$ \\
\hline Obese, $\mathrm{BMI} \geq 30$ & $44(29.3)$ & $1.06(0.45,2.51)$ & $1.54(0.58,4.11)$ & $1.81(0.83,3.94)$ & $1.89(0.78,4.61)$ & $0.76(0.24,2.38)$ & $0.86(0.24,3.07)$ & $1.98(0.61,6.40)$ & $1.98(0.58,6.74)$ \\
\hline $\begin{array}{l}\text { Waist Circumference } \geq 94 \\
\text { (males), } \geq 80 \text { (females) cm }\end{array}$ & 49.3) & $0.87(0.37,2.09)$ & $1.40(0.51,3.83)$ & $1.61(0.72,3.62)$ & $2.00(0.76,5.27)$ & $0.47(0.15,1.50)$ & $0.54(0.15,1.95)$ & $1.51(0.46,4.99)$ & $1.47(0.43,5.00)$ \\
\hline Glucose $\geq 100$ mg/dL (fasting) & $51(34.0)$ & $3.25(1.48,7.12)$ & $3.95(1.63,9.58)$ & $2.08(1.03,4.21)$ & $1.69(0.77,3.68)$ & $2.97(1.03,8.57)$ & $4.10(1.15,14.59)$ & $1.96(0.68,5.67)$ & $1.96(0.64,5.98)$ \\
\hline LDL cholesterol $\geq 2.59 \mathrm{mmol} / \mathrm{L}$ & $101(67.3)$ & $0.64(0.29,1.39)$ & $0.68(0.29,1.60)$ & $1.38(0.67,2.82)$ & $0.86(0.37,1.97)$ & $0.46(0.16,1.36)$ & $0.39(0.12,1.28)$ & $2.09(0.73,5.99)$ & $1.76(0.59,5.18)$ \\
\hline Triglycerides $\geq 1.7 \mathrm{mmol} / \mathrm{L}$ & $45(30.0)$ & $0.12(0.05,0.31)$ & $0.09(0.03,0.25)$ & $0.40(0.18,0.86)$ & $0.39(0.16,0.95)$ & $0.57(0.20,1.63)$ & $0.52(0.17,1.61)$ & $1.34(0.45,4.02)$ & $1.17(0.36,3.77)$ \\
\hline $\begin{array}{r}\mathrm{HDL} \text { cholesterol }<1.03 \text { (males), } \\
<1.29 \text { (females) } \mathrm{mmol} / \mathrm{L}\end{array}$ & 47 (31.3) & $2.36(1.00,5.58)$ & $1.93(0.74,5.02)$ & $0.62(0.30,1.31)$ & $0.64(0.28,1.50)$ & $7.15(1.99,25.70)$ & $5.85(1.48,23.07)$ & $0.45(0.14,1.43)$ & $0.51(0.16,1.65)$ \\
\hline $\begin{array}{r}\text { Triglyceride/HDL ratio }>3.5 \\
\text { (males), }>2.5 \text { (females) }\end{array}$ & $55(36.7)$ & $0.14(0.05,0.44)$ & $0.14(0.04,0.48)$ & $0.43(0.16,1.15)$ & $0.53(0.18,1.55)$ & $0.49(0.16,1.52)$ & $0.56(0.16,1.94)$ & $1.28(0.39,4.25)$ & $1.15(0.31,4.32)$ \\
\hline C-reactive protein $\geq 10 \mathrm{mg} / \mathrm{L}$ & $43(28.7)$ & $0.15(0.06,0.39)$ & $0.13(0.04,0.36)$ & $0.20(0.08,0.49)$ & $0.28(0.10,0.75)$ & $0.38(0.13,1.12)$ & $0.45(0.14,1.44)$ & $1.21(0.39,3.74)$ & $1.14(0.33,3.98)$ \\
\hline Systolic $\geq 130 \mathrm{mmHg}$ & $24(16.0)$ & $1.50(0.56,4.03)$ & $1.69(0.55,5.15)$ & $1.12(0.5462 .76)$ & $0.85(0.30,2.38)$ & $0.36(0.09,1.39)$ & $0.38(0.08,1.81)$ & $2.21(0.54,9.04)$ & $2.73(0.56,13.18)$ \\
\hline Diastolic $\geq 85$ mmHg & $23(15.3)$ & $2.07(0.69,6.22)$ & $2.20(0.59,8.16)$ & $0.76(0.29,2.00)$ & $0.46(0.15,1.41)$ & $0.21(0.05,0.97)$ & $0.16(0.03,0.90)$ & $2.24(0.47,10.71)$ & $3.50(0.59,20.68)$ \\
\hline
\end{tabular}

Adjusted for age, sex, site location.

"Adjusted for age, sex, site location, marital status, education, paid employment, smoking, alcohol use, and fish intake. 
Table 6 Adjusted* associations between metals (dichotomized at median) and cardiometabolic risk factors, controlling for percent body fat (\%BF)

\begin{tabular}{|c|c|c|c|c|c|c|c|c|c|}
\hline \multirow[b]{3}{*}{ Cardiometabolic risk factor } & \multirow[b]{3}{*}{ N (\%) } & \multicolumn{2}{|c|}{ Lead $(>1.66 \mu \mathrm{g} / \mathrm{dL})$} & \multicolumn{2}{|c|}{ Cadmium (>0.008 $\mu \mathrm{g} / \mathrm{L})$} & \multicolumn{2}{|c|}{ Arsenic (>8.48 $\mu \mathrm{g} / \mathrm{L})$} & \multicolumn{2}{|c|}{ Mercury $(>1.49 \mu \mathrm{g} / \mathrm{L})$} \\
\hline & & $\begin{array}{l}\text { Core model } \\
\text { plus } \% \mathrm{BF}\end{array}$ & $\begin{array}{l}\text { Fully-adjusted } \\
\text { plus \%BF }\end{array}$ & $\begin{array}{l}\text { Core model } \\
\text { plus } \% \mathrm{BF}\end{array}$ & $\begin{array}{l}\text { Fully-adjusted } \\
\text { plus } \% B F\end{array}$ & $\begin{array}{l}\text { Core model } \\
\text { plus } \% \mathrm{BF}\end{array}$ & $\begin{array}{l}\text { Fully-adjusted } \\
\text { plus } \% \mathrm{BF}\end{array}$ & $\begin{array}{l}\text { Core model } \\
\text { plus } \% \mathrm{BF}\end{array}$ & $\begin{array}{l}\text { Fully-adjusted } \\
\text { plus } \% \mathrm{BF}\end{array}$ \\
\hline & & OR $(95 \% \mathrm{Cl})^{*}$ & OR $(95 \% \mathrm{Cl})^{* *}$ & OR $(95 \% \mathrm{Cl})^{*}$ & OR $(95 \% \mathrm{Cl})^{* *}$ & OR $(95 \% \mathrm{Cl})^{*}$ & OR $(95 \% \mathrm{Cl})^{* *}$ & OR $(95 \% \mathrm{Cl})^{*}$ & OR $(95 \% \mathrm{CI})^{* *}$ \\
\hline Overweight, $\mathrm{BMI} \geq 25$ & $74(49.3)$ & $0.90(0.34,2.40)$ & $0.88(0.31,2.51)$ & $0.55(0.23,1.33)$ & $0.44(0.17,1.15)$ & $0.48(0.12,1.87)$ & $0.36(0.08,1.61)$ & $3.35(0.82,13.67)$ & $3.24(0.80,13.12)$ \\
\hline Obese, $\mathrm{BMI} \geq 30$ & $44(29.3)$ & $2.33(0.73,7.43)$ & $2.70(0.75,9.75)$ & $2.04(0.78,5.33)$ & $1.99(0.64,6.12)$ & $1.68(0.37,7.72)$ & $1.43(0.28,7.23)$ & $3.37(0.67,16.86)$ & $4.18(0.82,21.31)$ \\
\hline $\begin{array}{l}\text { Waist Circumference } \geq 94 \mathrm{~cm} \\
\text { (males) or } \geq 80 \mathrm{~cm} \text { (females) }\end{array}$ & $74(49.3)$ & $2.23(0.68,7.24)$ & $4.53(1.06,19.48)$ & $1.09(0.40,2.95)$ & $1.40(0.45,4.42)$ & $1.19(0.25,5.60)$ & $2.39(0.38,15.11)$ & $2.91(0.55,15.40)$ & $2.76(0.52,14.76)$ \\
\hline Glucose $\geq 100 \mathrm{mg} / \mathrm{dL}$ (fasting) & $51(34.0)$ & $4.82(2.01,11.53)$ & $4.99(1.97,12.69)$ & $2.01(0.98,4.13)$ & $1.60(0.72,3.56)$ & $4.81(1.48,15.58)$ & $5.62(1.49,21.19)$ & $2.12(0.71,6.33)$ & $2.10(0.66,6.64)$ \\
\hline LDL cholesterol $\geq 2.59 \mathrm{mmol} / \mathrm{L}$ & $101(67.3)$ & $0.78(0.35,1.76)$ & $0.77(0.32,1.86)$ & $1.25(0.60,2.62)$ & $0.75(0.32,1.76)$ & $0.64(0.21,1.94)$ & $0.50(0.15,1.71)$ & $2.15(0.73,6.33)$ & $1.78(0.58,5.51)$ \\
\hline Triglycerides $\geq 1.7 \mathrm{mmol} / \mathrm{L}$ & $45(30.0)$ & $0.12(0.05,0.32)$ & $0.09(0.03,0.26)$ & $0.38(0.17,0.83)$ & $0.36(0.15,0.90)$ & $0.63(0.22,1.84)$ & $0.58(0.18,1.84)$ & $1.37(0.45,4.14)$ & $1.22(0.38,3.93)$ \\
\hline $\begin{array}{l}\mathrm{HDL} \text { cholesterol }<1.03 \text { (males) } \\
\quad \text { or }<1.29 \text { (females) } \mathrm{mmol} / \mathrm{L}\end{array}$ & $47(31.3)$ & $2.64(1.08,6.50)$ & $2.10(0.79,5.61)$ & $0.60(0.29,1.2)$ & $0.61(0.26,1.44)$ & $9.81(2.41,39.88)$ & $8.02(1.84,35.01)$ & $0.44(0.14,1.41)$ & $0.50(0.15,1.63)$ \\
\hline $\begin{array}{r}\text { Triglyceride/HDL ratio }>3.5 \\
\text { (males) or }>2.5 \text { (females) }\end{array}$ & $55(36.7)$ & $0.14(0.04,0.43)$ & $0.15(0.04,0.51)$ & $0.42(0.16,1.13)$ & $0.53(0.18,1.53)$ & $0.51(0.6,1.62)$ & $0.61(0.17,2.15)$ & $1.31(0.39,4.36)$ & $1.19(0.32,4.49)$ \\
\hline C-reactive protein $\geq 10 \mathrm{mg} / \mathrm{L}$ & $43(28.7)$ & $0.15(0.06,0.39)$ & $0.13(0.05,0.38)$ & $0.19(0.08,0.47)$ & $0.27(0.10,0.73)$ & $0.38(0.12,1.17)$ & $0.50(0.16,1.62)$ & $1.22(0.39,3.79)$ & $1.14(0.33,3.96)$ \\
\hline Systolic $\geq 130 \mathrm{mmHg}$ & $24(16.0)$ & $1.67(0.60,4.64)$ & $1.77(0.57,5.45)$ & $1.10(0.45,2.70)$ & $0.80(0.28,2.28)$ & $0.38(0.10,1.48)$ & $0.38(0.08,1.82)$ & $2.27(0.55,9.38)$ & $2.79(0.57,13.68)$ \\
\hline Diastolic $\geq 85 \mathrm{mmHg}$ & $23(15.3)$ & $2.35(0.75,7.37)$ & $2.37(0.62,9.07)$ & $0.74(0.28,1.96)$ & $0.39(0.12,1.26)$ & $0.22(0.05,1.00)$ & $0.15(0.03,0.91)$ & $2.30(0.47,11.15)$ & $4.22(0.67,26.39)$ \\
\hline
\end{tabular}

Adjusted for age, sex, site location, and percent body fat (\%BF).

"Adjusted for age, sex, site location, marital status, education, paid employment, smoking, alcohol use, fish intake, and percent body fat (\%BF). 
windows of exposure, susceptible populations at risk, and biological mechanisms of effect.

It is unclear whether adiposity may be a mediator, modifier, or outcome as a result of exposure to environmental chemicals, therefore, we presented the main outcome analyses with and without adjustment for adiposity. In most cases, the observed associations were stronger after adjustment for percent body fat. Adipose tissue is now recognized as an important endocrine organ responsible for maintaining homeostasis or making physiological adaptations in response to dietary lipids, including increased inflammation and insulin resistance [53]. Adipocytes secrete substances that contribute to peripheral insulin resistance, including adiponectin, resistin, TNF-alpha and interleukin 6 which interfere with glucose metabolism and exert lipotoxic effects on pancreatic beta cells [54]. However, most studies use BMI which cannot distinguish between overall lean vs. fat mass or body fat distribution. Future analyses in the larger METS cohort over time will be aimed at trying to disentangle these complex associations.

Our study has several strengths including the wide geographic distribution and resultant exposure variability in our subpopulations. By design, there is also a wide distribution of factors related to social and economic development and the subpopulations are relatively homogeneous with respect to ancestral/genetic background [39]. African descent is a known risk factor for cardiometabolic diseases [55] and this study will allow us to further elucidate the potential for gene-environment interactions in this highrisk subgroup.

Despite these strengths, our study has several limitations: the small sample size of this preliminary study does not allow us to rule out chance findings or fully explore the role of adiposity as a potential mediator or modifier of these relationships. There may be selection bias given the unequal distribution of risk factors, such as sex and BMI, in the randomly chosen subset of individuals at baseline. It may be that cumulative lifetime exposure to one or more metals, not adequately captured by blood levels measured cross-sectionally in our study, are responsible for adverse health effects of exposure. Finally, we did not control for dietary factors, other than fish intake, or measure speciated arsenic or mercury levels in these analyses.

The role of diet both in the absorption, distribution, and metabolism of metals and in relation to the outcomes of interest in this study cannot be underestimated. The sites in our parent study were chosen specifically to "model the epidemiologic transition" occurring across five diverse countries of varying levels of social and economic development. The changing dietary and physical activity patterns worldwide are responsible for the "double burden" of malnutrition and obesity in many developing countries [56]. Thus, when examining the role of environmental contaminants, micronutrient status may be an important modifier of the effects of exposure, particularly to metals [57-59].

Health disparities in environmental exposures have also been widely documented [60] and environmental and occupational health risks may disproportionately associated with social disparities, such as lower socioeconomic status, due to co-occurring risks such as poor housing conditions, employment conditions, and nutritional deficits. African American race has been associated with elevated blood lead levels independent of age and socioeconomic conditions $[61,62]$ and, in fact, social disadvantage may further modify or exacerbate the adverse effects of environmental exposures [63]. The concept of allostatic load suggests that the cumulative dysregulation of biological systems due to changing social and environmental stressors may result in decreased effectiveness of adaptive responses and increased risk for disease [64]. Genetic susceptibility to metals exposure is also recognized [65]. Divalent metal transporter-1 (DMT1) functions in the transport of some divalent metal ions across the plasma membrane $[66,67]$ and racial-ethnic differences in DMT1 expression may underlie racial disparities in blood metals levels [68].

Health disparities in endocrine diseases have recently been reviewed by the Endocrine Society which issued a scientific policy statement calling for multilevel interventions to reduce disparities in diabetes and endocrine diseases [69]. Our data suggest that exposure to environmental contaminants should be considered when evaluating risk and designing prevention and intervention programs. Racial disparities in health cannot be explained solely on the basis of genetic susceptibility, poverty and access to care, lifestyle or environmental factors [70]. While the size of the effects of exposures to environmental contaminants may be relatively small, their impact on a population-level is large, given the widespread and everchanging distribution of environmental risk factors around the world and potential for cumulative effects from exposure to multiple pollutants simultaneously.

\section{Conclusions}

These data are suggestive of potentially important associations between blood metals concentrations and cardiometabolic risk. Insights about potential biological mechanisms underlying disparities in cardiometabolic health can be gained from exploring differences in environmental exposures. Research efforts should include environmental factors when studying the complex etiology of cardiometabolic diseases which could result in targeted interventions to decrease health disparities and the associated risks of ongoing exposures particularly in the developing world where environmental and occupational control policies may be lacking or inadequate to protect public health. 


\section{Abbreviations}

BMI: Body mass index; DMT-1: Divalent metal transporter-1; HDL: Highdensity lipoprotein; HDI: Human Development Index; $\mathrm{kg} / \mathrm{m}^{2}$ : Kilogram per meter-squared; LOD: Limit of detection; LDL: Low-density lipoprotein; METS: Modeling the Epidemiologic Transition Study; OR: Odds ratio; C.I.: Confidence interval; NHANES: National Health and Nutrition Examination Survey; NIST: National Institute of Standard and Technology; SRM: Standard Reference Material; $\mathrm{HNO}_{3}$ : Nitric acid; p: p-value; SD: Standard deviation; USA: United States of America.

\section{Competing interests}

The authors declare that they have no competing interests.

\section{Authors' contributions}

ASE conceived of the study with AL, conducted statistical analyses, and drafted the manuscript. AL provided access to the data and specimens, contributed to study design, statistical analyses, and drafting of the manuscript. PB and DS contributed to study design, statistical analyses, and drafting of the manuscript. LRD contributed to study design and coordinated data collection with site investigators: AL, PB, JPR, TEF, EVL. RADA and RSC contributed to study design and data collection. NL and JS were responsible for laboratory analyses and contributed to drafting the manuscript. All authors read and approved the final manuscript.

\section{Acknowledgements}

METS is funded in part by the National Institutes of Health (1R01DK80763). Laboratory analyses were supported in part by the National Institute of Environmental Health Sciences (NIEHS) through the Harvard NIEHS Center for Environmental Health (P30ES000002).

\section{Author details}

${ }^{1}$ Yale Center for Perinatal, Pediatric \& Environmental Epidemiology, Department of Chronic Disease Epidemiology, Yale School of Public Health, 1 Church St, New Haven, CT 06510, USA. ${ }^{2}$ Institute of Social and Preventive Medicine, Lausanne University Hospital, 10, route de la Corniche, Lausanne $\mathrm{CH}-1010$, Switzerland. ${ }^{3}$ Unit for the Prevention and Control of Cardiovascular Disease, Ministry of Health, Victoria, Mahe, Seychelles. ${ }^{4}$ Kwame Nkrumah University of Science and Technology, Kumasi, Ashanti, Ghana. ${ }^{5}$ St George's, University of London, London, UK. ${ }^{6}$ UWI Solutions for Developing Countries, University of the West Indies Mona, 25 West Road, Kingston 7, Jamaica. ${ }^{7}$ Research Unit for Exercise Science and Sports Medicine, Division of Health Sciences, University of Cape Town, Cape Town 7725, South Africa. ${ }^{8}$ Department of Environmental Health, Harvard School of Public Health, 677 Huntington Ave, Boston, MA 02115, USA. 'Department of Public Health Sciences, Stritch School of Medicine, Loyola University Chicago, 2160 S. First Ave, Maywood, IL 60153, USA.

Received: 31 March 2014 Accepted: 22 October 2014

Published: 5 November 2014

\section{References}

1. Kuo CC, Moon K, Thayer KA, Navas-Acien A: Environmental chemicals and type 2 diabetes: an updated systematic review of the epidemiologic evidence. Curr Diab Rep 2013, 13:831-849.

2. Thayer KA, Heindel JJ, Bucher JR, Gallo MA: Role of environmental chemicals in diabetes and obesity: a National Toxicology Program workshop review. Environ Health Perspect 2012, 120:779-789.

3. Zeliger HI: Lipophilic chemical exposure as a cause of cardiovascular disease. Interdiscip Toxicol 2013, 6:55-62.

4. Cowie CC, Rust KF, Byrd-Holt DD, Gregg EW, Ford ES, Geiss LS, Bainbridge KE, Fradkin JE: Prevalence of diabetes and high risk for diabetes using A1C criteria in the U.S. population in 1988-2006. Diabetes Care 2010, 33:562-568.

5. Ogden CL, Carroll MD, Curtin LR, McDowell MA, Tabak CJ, Flegal KM: Prevalence of overweight and obesity in the United States, 1999-2004. JAMA 2006, 295:1549-1555.

6. Flegal KM, Carroll MD, Ogden CL, Curtin LR: Prevalence and trends in obesity among US adults, 1999-2008. JAMA 2010, 303:235-241.

7. Ogden $\mathrm{CL}$, Carroll MD, Kit BK, Flegal KM: Prevalence of childhood and adult obesity in the United States, 2011-2012. JAMA 2014, 311:806-814.

8. Seidell JC: Obesity, insulin resistance and diabetes-a worldwide epidemic. Br J Nutr 2000, Suppl 1:S5-S8.
9. Baillie-Hamilton PF: Chemical toxins: a hypothesis to explain the global obesity epidemic. J Altern Complement Med 2002, 8:185-192.

10. Grün F, Blumberg B: Endocrine disrupters as obesogens. Mol Cell Endocrinol 2009, 304:19-29.

11. Longnecker MP, Daniels JL: Environmental contaminants as etiologic factors for diabetes. Environ Health Perspect 2001, 109:871-876.

12. Hyman MA: Environmental toxins, obesity, and diabetes: an emerging risk factor. Altern Ther Health Med 2010, 16:56-58.

13. Hu H: Exposure to metals. Prim Care 2000, 27:983-996

14. Järup L: Hazards of heavy metal contamination. Br Med Bull 2003, 68:167-182

15. Dyer CA: Heavy metals as endocrine-disrupting chemicals. In EndocrineDisrupting Chemicals: From Basic Science to Clinical Practice. Edited by Gore AC. Totowa, NJ: Humana Press Inc; 2007.

16. lavicoli I, Fontana L, Bergamaschi A: The effects of metals as endocrine disruptors. J Toxicol Environ Health B Crit Rev 2009, 12:206-223.

17. Tseng $\mathrm{CH}$ : The potential biological mechanisms of arsenic-induced diabetes mellitus. Toxicol Appl Pharmacol 2004, 197:67-83.

18. Chen YW, Yang CY, Huang CF, Hung DZ, Leung YM, Liu SH: Heavy metals, islet function and diabetes development. Islets 2009, 1:169-176.

19. Watson WH, Yager JD: Arsenic: extension of its endocrine disruption potential to interference with estrogen receptor-mediated signaling. Toxicol Sci 2007, 98:1-4.

20. Kaltreider RC, Davis AM, Lariviere JP, Hamilton JW: Arsenic alters the function of the glucocorticoid receptor as a transcription factor. Environ Health Perspect 2001, 109:245-251.

21. Alissa EM, Ferns GA: Heavy metal poisoning and cardiovascular disease. J Toxicol 2011, 2011:870125. doi: 10.1155/2011/870125. Epub 2011 Sep 8.

22. Jomova K, Valko M: Advances in metal-induced oxidative stress and human disease. Toxicology 2011, 283:65-87.

23. Vaziri ND: Mechanisms of lead-induced hypertension and cardiovascular disease. Am J Physiol Heart Circ Physiol 2008, 295:H454-H465.

24. Moon K, Guallar E, Navas-Acien A: Arsenic exposure and cardiovascular disease: an updated systematic review. Curr Atheroscler Rep 2012, 14:542-555.

25. Navas-Acien A, Guallar E, Silbergeld EK, Rothenberg SJ: Lead exposure and cardiovascular disease-a systematic review. Environ Health Perspect 2007, 115:472-482.

26. Tellez-Plaza M, Jones MR, Dominguez-Lucas A, Guallar E, Navas-Acien A: Cadmium exposure and clinical cardiovascular disease: a systematic review. Curr Atheroscler Rep 2013, 15:356.

27. Virtanen JK, Rissanen TH, Voutilainen S, Tuomainen TP: Mercury as a risk factor for cardiovascular diseases. J Nutr Biochem 2007, 18:75-85.

28. Lee BK, Kim Y: Blood cadmium, mercury, and lead and metabolic syndrome in South Korea: 2005-2010 Korean National Health and Nutrition Examination Survey. Am J Ind Med 2013, 56:682-692.

29. Moon SS: Additive effect of heavy metals on metabolic syndrome in the Korean population: the Korea National Health and Nutrition Examination Survey (KNHANES) 2009-2010. Endocrine 2014, 46:263-271.

30. Eckel RH, Grundy SM, Zimmet PZ: The metabolic syndrome. Lancet 2005, 365:1415-1428.

31. Friedrich MJ: Studies probe mechanisms that have a role in obesityassociated morbidities. JAMA 2012, 308:1077-1079.

32. Poirier P, Giles TD, Bray GA, Hong Y, Stern JS, Pi-Sunyer FX, Eckel RH, American Heart Association, Obesity Committee of the Council on Nutrition, Physical Activity, and Metabolism: Obesity and cardiovascular disease: pathophysiology, evaluation, and effect of weight loss: an update of the 1997 American Heart Association Scientific Statement on Obesity and Heart Disease from the Obesity Committee of the Council on Nutrition, Physical Activity, and Metabolism. Circulation 2006, 113:898-918.

33. Eyre H, Kahn R, Robertson RM: Preventing cancer, cardiovascular disease, and diabetes: a common agenda for the American Cancer Society, the American Diabetes Association, and the American Heart Association. CA Cancer J Clin 2004, 54:190-207.

34. Wildman RP, Muntner P, Reynolds K, McGinn AP, Rajpathak S, Wylie-Rosett J Sowers MR: The obese without cardiometabolic risk factor clustering and the normal weight with cardiometabolic risk factor clustering: prevalence and correlates of 2 phenotypes among the US population (NHANES 1999-2004). Arch Intern Med 2008, 168:1617-1624.

35. Ortega FB, Lee DC, Katzmarzyk PT, Ruiz JR, Sui X, Church TS, Blair SN: The intriguing metabolically healthy but obese phenotype: cardiovascular prognosis and role of fitness. Eur Heart J 2013, 34:389-397. 
36. Hamer M, Stamatakis E: Metabolically healthy obesity and risk of all-cause and cardiovascular disease mortality. J Clin Endocrinol Metab 2012, 97:2482-2488

37. Hankinson AL, Daviglus ML, Van Horn L, Chan Q, Brown I, Holmes E, Elliott P, Stamler J: Diet composition and activity level of at risk and metabolically healthy obese American adults. Obesity 2013, 21:637-643.

38. Kramer CK, Zinman B, Retnakaran R: Are metabolically healthy overweight and obesity benign conditions? A systematic review and meta-analysis. Ann Intern Med 2013, 159:758-769.

39. Luke A, Bovet P, Forrester TE, Lambert EV, Plange-Rhule J, Schoeller DA, Dugas LR, Durazo-Arvizu RA, Shoham D, Cooper RS, Brage S, Ekelund U, Steyn NP: Protocol for the modeling the epidemiologic transition study: a longitudinal observational study of energy balance and change in body weight, diabetes and cardiovascular disease risk. BMC Public Health 2011, 11:927.

40. United Nations Development Programme (UNDP): 2011 Human Development Report-Sustainability and Equity: A Better Future for All. [http://www.undp.org/ content/undp/en/home/librarypage/hdr/human_developmentreport2011/]

41. Armstrong T, Bull F: Development of the World Health Organization Global Physical Activity Questionnaire (GPAQ). J Public Health 2006, 14:66-70.

42. World Health Organization (WHO): Global Physical Activity Questionnaire. [http://www.who.int/chp/steps/GPAQ/en/]

43. The World Bank Group: Core Welfare Indictor Questionnaire (CWIQ). [http://go.worldbank.org/66ELZUGJ30]

44. Huang PL: A comprehensive definition for metabolic syndrome. Dis Model Mech 2009, 2:231-237.

45. International Diabetes Federation (IDF): The IDF Consensus Worldwide Definition of Metabolic Syndrome. [http://www.idf.org/publications/idfconsensus-definition-metabolic-syndrome]

46. Centers for Disease Control and Prevention (CDC): Fourth National Report on Human Exposure to Environmental Chemicals-Updated Tables, September 2013. [http://www.cdc.gov/exposurereport]

47. Navas-Acien A, Silbergeld EK, Pastor-Barriuso R, Guallar E: Arsenic exposure and prevalence of type 2 diabetes in US adults. JAMA 2008, 300:814-822

48. Schwartz $G G$, II'yasova D, Ivanova A: Urinary cadmium, impaired fasting glucose, and diabetes in the NHANES III. Diabetes Care 2003, 26:468-470.

49. Schug TT, Janesick A, Blumberg B, Heindel JJ: Endocrine disrupting chemicals and disease susceptibility. J Steroid Biochem Mol Biol 2011, 127:204-215.

50. Gluckman PD, Hanson MA, Pinal C: The developmental origins of adult disease. Matern Child Nutr 2005, 1:130-141.

51. Newbold RR, Padilla-Banks E, Jefferson WN: Environmental estrogens and obesity. Mol Cell Endocrinol 2009, 304:84-89.

52. Heindel JJ, vom Saal FS: Role of nutrition and environmental endocrine disrupting chemicals during the perinatal period on the aetiology of obesity. Mol Cell Endocrinol 2009, 304:90-96.

53. Galic S, Oakhill JS, Steinberg GR: Adipose tissue as an endocrine organ. Mol Cell Endocrinol 2010, 316:129-139.

54. Keller U: From obesity to diabetes. Int J Vitam Nutr Res 2006, 76:172-177.

55. Kurian AK, Cardarelli KM: Racial and ethnic differences in cardiovascular disease risk factors: a systematic review. Ethn Dis 2007, 17:143-152.

56. Food and Agriculture Organization (FAO): The double burden of malnutrition: case studies from six developing countries. [http://www.fao. org/ag/magazine/0602sp1.htm]

57. Goyer RA: Nutrition and metal toxicity. Am J Clin Nutr 1995, 61:646S-650S.

58. Peraza MA, Ayala-Fierro F, Barber DS, Casarez E, Rael LT: Effects of micronutrients on metal toxicity. Environ Health Perspect 1998, 106:203-216.

59. Sandström B: Micronutrient interactions: effects on absorption and bioavailability. Br J Nutr 2001, 85:S181-S185.

60. Gee GC, Payne-Sturges DC: Environmental health disparities: a framework integrating psychosocial and environmental concepts. Environ Health Perspect 2004, 112:1645-1653.

61. Pirkle JL, Brody DJ, Gunter EW, Kramer RA, Paschal DC, Flegal KM, Matte TD: The decline in blood lead levels in the United States. The National Health and Nutrition Examination Surveys (NHANES). JAMA 1994, 272:284-291.

62. Muntner $P$, Menke A, DeSalvo KB, Rabito FA, Batuman V: Continued decline in blood lead levels among adults in the United States: the National Health and Nutrition Examination Surveys. Arch Intern Med 2005, 165:2155-2161.
63. Hicken MT, Gee GC, Morenoff J, Connell CM, Snow RC, Hu H: A novel look at racial health disparities: the interaction between social disadvantage and environmental health. Am J Public Health 2012, 102:2344-2351.

64. Beckie TM: A systematic review of allostatic load, health, and health disparities. Biol Res Nurs 2012, 14:311-346.

65. Onalaja AO, Claudio L: Genetic susceptibility to lead poisoning Environ Health Perspect 2000, 108:23-28.

66. Garrick MD, Dolan KG, Horbinski C, Ghio AJ, Higgins D, Porubcin M, Moore EG, Hainsworth LN, Umbreit JN, Conrad ME, Feng L, Lis A, Roth JA, Singleton S, Garrick LM: DMT1: a mammalian transporter for multiple metals. Biometals $2003,16: 41-54$

67. Mims MP, Prchal JT: Divalent metal transporter 1. Hematology 2005, 10:339-345.

68. Ngueta G: Racial disparities in children's blood lead levels: possible implication of divalent metal transporter 1. Med Hypotheses 2014, 82:71-73.

69. Golden SH, Brown A, Cauley JA, Chin MH, Gary-Webb TL, Kim C, Sosa JA,

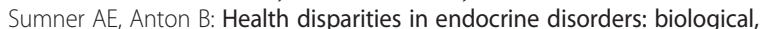
clinical, and nonclinical factors-an Endocrine Society scientific statement. J Clin Endocrinol Metab 2012, 97:E1579-E1639.

70. Olden K, White SL: Health-related disparities: influence of environmental factors. Med Clin North Am 2005, 89:721-738.

\section{doi:10.1186/1476-069X-13-90}

Cite this article as: Ettinger et al:: Distribution of metals exposure and associations with cardiometabolic risk factors in the "Modeling the Epidemiologic Transition Study". Environmental Health 2014 13:90.

\section{Submit your next manuscript to BioMed Central and take full advantage of:}

- Convenient online submission

- Thorough peer review

- No space constraints or color figure charges

- Immediate publication on acceptance

- Inclusion in PubMed, CAS, Scopus and Google Scholar

- Research which is freely available for redistribution
C BioMed Central 